\title{
ACKNOWLEDGEMENT TO REFEREES
}

(c) Springer Nature Switzerland AG 2019

Dear Reader,

As we reach the final issue of CNS Drugs for 2019, we wish to reflect on another successful year's achievements, and to thank all those who have contributed their time and effort to guarantee the high quality of our content.

2019 was a successful year for CNS Drugs. Over 75 articles have been published; the most popular of these, in terms of downloads from SpringerLink, have been:

- Wheless et al. Pharmacokinetics and Tolerability of Multiple Doses of Pharmaceutical-Grade Synthetic Cannabidiol in Pediatric Patients with Treatment-Resistant Epilepsy. (https://link.springer.com/article/10.1007/s40263-019-00624-4)

- Pladevall-Vila et al. Risk of Acute Liver Injury in Agomelatine and Other Antidepressant Users in Four European Countries: A Cohort and Nested Case-Control Study Using Automated Health Data Sources (https://link.springer.com/artic le/10.1007/s40263-019-00611-9)

- Green et al. Novel Treatment Opportunities Against Cognitive Impairment in Parkinson's Disease with an Emphasis on Diabetes-Related Pathways (https://link.springer.com/article/10.1007/s40263-018-0601-x)

- Kohlschütter et al. Current and Emerging Treatment Strategies for Neuronal Ceroid Lipofuscinoses (https://link.sprin ger.com/article/10.1007/s40263-019-00620-8)

- Van Weering \& Scheper. Endolysosome and Autolysosome Dysfunction in Alzheimer's Disease: Where Intracellular and Extracellular Meet (https://link.springer.com/article/10.1007/s40263-019-00643-1)

- Hobart et al. Assessment of Clinically Meaningful Improvements in Self-Reported Walking Ability in Participants with Multiple Sclerosis: Results from the Randomized, Double-Blind, Phase III ENHANCE Trial of Prolonged-Release Fampridine (https://link.springer.com/article/10.1007/s40263-018-0586-5)

- Lipton et al. DFN-02, Sumatriptan $10 \mathrm{mg}$ Nasal Spray with Permeation Enhancer, for the Acute Treatment of Migraine: A Randomized, Double-Blind, Placebo-Controlled Study Assessing Functional Disability and Subject Satisfaction with Treatment (https://link.springer.com/article/10.1007/s40263-019-00614-6)

- Xu et al. A Multi-Center, Open-Label, Pharmacokinetic Drug Interaction Study of Erenumab and a Combined Oral Contraceptive in Healthy Females (https://link.springer.com/article/10.1007/s40263-019-00626-2)

- Frieder et al. Pharmacotherapy of Postpartum Depression: Current Approaches and Novel Drug Development (https:// link.springer.com/article/10.1007/s40263-019-00605-7)

- Wang et al. Combination Therapy with Dipyridamole and Clopidogrel for Secondary Stroke Prevention in AspirinIntolerant Patients After Myocardial Infarction: Results of a Nationwide Case-Control Study (https://link.springer.com/ article/10.1007/s40263-018-0591-8).

Social media sharing of information has become important and is another metric with which to measure the reach of articles. This year the following articles published in CNS Drugs scored highly on the Altmetric system:

- Jafferany \& Patel. Skin-Picking Disorder: A Guide to Diagnosis and Management (https://link.springer.com/artic le/10.1007/s40263-019-00621-7)

- McAlary et al. Emerging Developments in Targeting Proteotoxicity in Neurodegenerative Diseases (https://link.springer. com/article/10.1007\%2Fs40263-019-00657-9) 
- Bergsland et al. Targeting Iron Dyshomeostasis for Treatment of Neurodegenerative Disorders (https://link.springer.com/ article/10.1007\%2Fs40263-019-00668-6)

- Lanzillotta et al. Targeting Mitochondria in Alzheimer Disease: Rationale and Perspectives (https://link.springer.com/ article/10.1007\%2Fs40263-019-00658-8)

- Artukoglu \& Bloch. The Potential of Cannabinoid-Based Treatments in Tourette Syndrome (https://link.springer.com/ article/10.1007\%2Fs40263-019-00627-1)

The quality of articles published in the journal is also reflected in its most recent impact factor of 4.192. This places $C N S$ Drugs in the top quartile of the Pharmacology and Pharmacy, Psychiatry, and Clinical Neurology categories.

We thank the authors who have contributed articles to CNS Drugs over the course of 2019. The skill and dedication of all authors are critical to the continued publication of the journal. The quality of published articles is also testament to the significant efforts of the peer reviewers, whose commitment ensures that the journal's content is held to the highest possible standard. We would like to thank the following individuals who acted as reviewers for CNS Drugs in the last 12 months:

Jan Aaseth, Norway

Umberto Aguglia, Italy

Sura Alwan, Canada

Gail D. Anderson, USA

Angelo Antonini, Italy

Josephine Arendt, UK

Vicent Balanzá, Spain

Ettore Beghi, Italy

Chantal Berna, Switzerland

Frank M.C. Besag, UK

Isabelle Beuchat, Switzerland

Pavan Bhargava, USA

Robert Bies, USA

Sophie Billioti de Gage, France

Steven Black, USA

Richard K. Bogan, USA

Vincent Bonhomme, Belgium

Laura Jayne Bonnett, UK

Margherita Brindisi, USA

Rebecca Burch, USA

Joseph R. Calabrese, USA

Craig Campbell, Canada

Ann Catherine Childress, USA

Catherine Chiron, France

Maria Roberta Cilio, USA

Andrea Cippitelli, USA

Leslie Citrome, USA

Jeffrey A. Cohen, USA

Elizabeth Regina Comini Frota, Brazil

Veronica Contini, Brazil

Constanza J. Cortes, USA

Patricia K. Coyle, USA

Julie L. Cunningham, USA

Marja-Liisa Dahl, Sweden

Yves Dauvilliers, France

Erica De Candia, Italy

Simone Di Giovanni, UK

Katherine Donegan, UK
Gail D'Onofrio, USA

Gilles Edan, France

Lars Edvinsson, Sweden

Jorgen Ekstrom, Sweden

Britta Engelhardt, Switzerland

Sophie Erhardt, Sweden

Robert Erickson, USA

Athanasios Evangeliou, Greece

Edward Faught, USA

Javier Fernandez-Ruiz, Spain

Martha Feucht, Austria

Massimo Filippi, Italy

Thomas Foltynie, UK

Sarah N. Fontaine, USA

Diego Fornasari, Italy

Mark Steven Freedman, Canada

Frederick G. Freitag, USA

Adeline Gallini, France

Cassandra Gipson-Reichardt, USA

Jennifer Glaus, Switzerland

Ana Gonzalez-Pinto, Spain

William W.V. Good, USA

David J. Greenblatt, USA

P. Grewe, Germany

Carolyn Goldschmidt, USA

Ewout J.N. Groen, UK

Christopher Grunseich, USA

Heinz C.R. Grunze, UK

Carolina L. Haass-Koffler, USA

Angelos Halaris, USA

Simon Hametner, Austria

Tor-Arne Hegvik, Norway

Sean Hennessy, USA

Nathan Herrmann, Canada

Morten Hesse, Denmark

Ellen Astrid Holm, Denmark

Christian Holscher, UK

Martin Holtkamp, Germany 
David Houghton, USA

Jason H. Huang, USA

Samuel F. Hunter, USA

Yushi Inoue, Japan

Md. Jakaria, Republic of Korea

Piotr Janik, Poland

Kurt Jellinger, Austria

Misty Rayna Jenkins, Australia

Jukka Jokinen, Finland

Meldon Kahan, Canada

Siegfried Kasper, Austria

Joseph Shimon Kass, USA

Jatinder Katyal, India

Regina Katzenschlager, Austria

Nicholas A. Keks, Australia

Christoph Kellinghaus, Germany

Lauren E. Kelly, Canada

David Kennaway, Australia

Rita Khoury, USA

Thomas Klopstock, Germany

Roger Knaggs, UK

$Y u$ Ko, Taiwan

Ole Kohler-Forsberg, Denmark

Giedre Krenciute, USA

Joji B. Kuramatsu, Germany

Jose L. Labandeira-Garcia, Spain

H. Lamberink, the Netherlands

Christian Lampl, Austria

Maryse Lapeyre-Mestre, France

Simona Lattanzi, Italy

Patricia Lavand'homme, Belgium

John A. Lawson, Australia

Bernard Le Foll, Canada

Mary Lee, USA

Vladimir V. Lerner, Israel

Andrew P. Lieberman, USA

Michael Linnebank, Germany

Ryan W. Logan, USA

Yoon Kong Loke, UK

Hendrika Luijendijk, the Netherlands

Gin S. Malhi, Australia

Wai Hong Man, the Netherlands

Ajay Manhapra, USA

Lars Hфj Markvardsen, Denmark

Paolo P. Martelletti, Italy

Andrea Mastinu, Italy

Massimo Carlo Mauri, Italy

Donovan T. Maust, USA

Amelia J. McFarland, Australia

Patrizia Mecocci, Italy

Christoph R. Meier, Switzerland

Guiseppe Raffaele Micieli, Italy

Laurent Misery, France
Ram K. Mishra, Canada

Sara Mole, UK

Paula I. Moreira, Portugal

Marco Mula, UK

Thomas Muller, Germany

Kirsten Muller-Vahl, Germany

Kassandra Munger, USA

Simon S. Murray, Australia

Henry A. Nasrallah, USA

Atsushi Natsume, Japan

Roland Nau, Germany

Zsofia Nemoda, Hungary

Sarah Nevitt, UK

Huu Phuc Nguyen, Germany

Bobby Nossaman, USA

Brian O'Donoghue, Australia

Patricia Ohrmann, Germany

William G. Ondo, USA

Lin Kooi Ong, Australia

Asher Ornoy, Israel

Kristi R. Overgaard, Norway

Christian Aavang Ovesen, Denmark

Rajesh Pahwa, USA

Antoine Pariente, France

Ajay K. Parsaik, USA

Jennifer Payne, USA

Santiago Perez-Lloret, Argentina

Merce Pineda, Spain

Michael S. Rafii, USA

Nicolas Ramoz, France

Hemachandra P.H. Reddy, USA

Maria A. Rocca, Italy

Paul Rolan, Australia

Adriane R. Rosa, Brazil

Emilio Russo, Italy

Juan M. Saavedra, USA

Rajesh C. Sachdeo, USA

Jacqueline Sagen, USA

Marko Sahinovic, the Netherlands

Martha Sajatovic, USA

Ivan Sanchez Fernandez, USA

Gabriele Sani, Italy

Jerome Sarris, Australia

Jana J. Sawynok, Canada

Andrew J. Saxon, USA

Georgios Schoretsanitis, Switzerland

Daniel Schottle, Germany

Wilhelm W. Schulte-Mattler, Germany

Mehmet Güney Şenol, Turkey

David M. Simpson, USA

Harvey S. Singer, USA

Sarah Snider, USA

Mehmet M. Sofuoglu, USA 
Amber Southwell, USA

Michael Soyka, Germany

Sade Spencer, USA

Edoardo Spina, Italy

Mark A. Stein, USA

Fabrizio Stocchi, Italy

Claudia Stollberger, Austria

Adam Strzelczyk, Germany

Norio Sugawara, Japan

James M. Swanson, USA

Erik R. Swenson, USA

Rumiko Takayama, Japan

Andrew M. Tan, USA

Bruce Taylor, Australia

Mishka Terplan, USA

Giuseppina Tesco, USA

Georgios Tsivgoulis, Greece

Marco Tuccori, Italy
Guillaume van Niel, France

Alfredo Vannacci, Italy

Christopher W. Vaughan, Australia

Eduard Vieta, Spain

Venugopal Y. Vishnu, India

Ladislav Volicer, USA

Mark S. Wainwright, USA

Christopher Walker, New Zealand

Dominic Walsh, USA

Mark Walterfang, Australia

Christopher A. Wassif, USA

Jill Weimer, USA

Margaret D. Weiss, USA

Heinz Wiendl, Germany

John Wild, UK

Katie Witkiewitz, USA

Zhengping Zhuang, USA

In addition, we would like to thank the members of the journal's Honorary Editorial Board, who have acted as peer reviewers and authors, and have provided guidance on journal content, policy and processes:

David S. Baldwin, University of Southampton, Southampton, England

Robert L. Barkin, Rush University, Chicago, IL, USA

Thomas R.E. Barnes, Imperial College London, London, England

Michael Berk, Deakin University, Geelong, VIC, Australia

Meir Bialer, The Hebrew University of Jerusalem, Jerusalem, Israel

Jose Biller, Loyola University Chicago, Maywood, IL, USA

Henry Brodaty, Prince of Wales Hospital, Randwick, NSW, Australia

Martin J. Brodie, West Glasgow Ambulatory Care Hospital, Glasgow, Scotland

Matthew J. Carpenter, Medical University of South Carolina, Charleston, SC, USA

Marc C. Chamberlain, University of Washington, Seattle, WA, USA

David R. Coghill, University of Melbourne, Melbourne, VIC, Australia

Samuel Cortese, University of Southampton, Southampton, England

Mervyn J. Eadie, University of Queensland, Brisbane, QLD, Australia

Martin R. Farlow, Indiana University School of Medicine, Indianapolis, IN, USA

Giovanni A. Fava, University of Bologna, Bologna, Italy

Susan H. Fox, University Health Network, Toronto, ON, Canada

George T. Grossberg, St Louis University School of Medicine, St. Louis, MO, USA

Christian Guilleminault, Stanford Health Services, Stanford, CA, USA

David Gurwitz, Tel-Aviv University, Tel Aviv, Israel

Martina Hahn, Vitos Klinik, Eltville, Germany

Göran Hajak, University of Regensburg, Regensburg, Germany

Hans-Peter Hartung, Heinrich-Heine-University, Duesseldorf, Germany

Michael W. Jann, University of North Texas, Fort Worth, TX, USA

David Kemp, Case Western Reserve University, Cleveland, OH, USA

Susan G. Kornstein, Virginia Commonwealth University, Richmond, VA, USA

Patrick Kwan, University of Melbourne, Royal Melbourne Hospital, Parkville, VIC, Australia

Raymond W. Lam, University of British Columbia, Vancouver, BC, Canada

Lorenzo Leggio, NIAAA and NIDA, NIH, Bethesda, MD, USA

Brian E. Leonard, National University of Ireland, Galway, Ireland

Philip Mitchell, University of New South Wales, Randwick, NSW, Australia

Jeffrey H. Newcorn, Icahn School of Medicine at Mount Sinai, New York, NY, USA 
Emilio Perucca, University of Pavia, Pavia, Italy

Robert M. Post, George Washington University, Washington DC and Bipolar Collaborative Network, Washington DC, USA Samir K. Praharaj, Kasturba Medical College, Manipal, Karnataka, India

Alan M. Rapoport, University of California, Los Angeles, CA, USA

Olivier Rascol, University Hospital, Toulouse, France

Dennis A. Revicki, Evidera, Bethesda, MD, USA

Karen L. Roos, Indiana University Hospital, Indianapolis, IN, USA

Jerrold F. Rosenbaum, Massachusetts General Hospital, Boston, MA, USA

Matthew V. Rudorfer, National Institute of Mental Health, Bethesda, MD, USA

Steven C. Schachter, Beth Israel Deaconess Medical Center, Boston, MA, USA

Stephan A. Schug, Royal Perth Hospital, Perth, WA, Australia

Soraya S. Seedat, Stellenbosch University, Cape Town, South Africa

Stephen D. Silberstein, Thomas Jefferson University Hospital, Philadelphia, PA, USA

David Taylor, Maudsley Hospital, London, England

Claudia Trenkwalder, Paracelsus-Elena Hospital, Kassel, Germany

Eugen Trinka, Paracelsus Medical University Salzburg, Salzburg, Austria

Benedetto Vitiello, University of Turin, Turin, Italy

Bianca Weinstock-Guttman, State University of New York, Buffalo, NY, USA

John W. Winkelman, Harvard Medical School, Boston, MA, USA

Ian C.K. Wong, University of Hong Kong, Hong Kong

John Zajecka, Rush University Medical Center, Chicago, IL, USA

We do hope that you have found the articles published throughout the year in CNS Drugs to be interesting and informative. The editorial schedule for 2020 is well under way, and we are looking forward to bringing you many high-quality and authoritative articles over the coming year.

With best wishes,

Kate Palmer \& Sue Pochon 